Check for updates

Cite this: RSC Adv., 2017, 7, 55720

Received 25th September 2017 Accepted 1st December 2017

DOI: 10.1039/c7ra10598c

rsc.li/rsc-advances

\section{Photo-triggered enzymatic degradation of biodegradable polymers $\uparrow$}

\author{
Yoshihiro Kikkawa, (D) Satoko Tanaka and Yasuo Norikane* \\ A material whose enzymatic degradation is initiated by an external stimulus is of great importance from the \\ viewpoint of practical biodegradable polymers. In this context, we developed a method that controls the \\ initiation of enzymatic degradation by light. 4,4'-Didecyloxy-3-methylazobenzene (Azo), which melts \\ upon exposure to UV radiation, was coated onto biodegradable poly(L-lactide) and the enzymatic \\ degradation of the polymer by proteinase $\mathrm{K}$ was successfully initiated and controlled by tuning the \\ solid-liquid transition of the azo-based compound. This stimulus-triggered enzymatic degradation is \\ a significant contribution to the development of lifetime-controlled biodegradable materials.
}

\section{Introduction}

Biodegradable polymers are attracting increasing attention as environmentally friendly eco-materials produced from renewable biomass. ${ }^{1}$ Their biodegradation is initiated by the action of hydrolytic enzymes secreted by microorganisms. ${ }^{2}$ Ideal biodegradable polymers should maintain their functions as polymeric materials during use, but begin to degrade shortly after disposal. Therefore, controlling enzymatic degradation is a fundamental issue for the effective and practical use of such biodegradable materials. ${ }^{2}$ Controlling the enzymatic degradation rate (Fig. 1A) has been achieved by copolymerization, chemical modification, and blending of biodegradable polymers. $^{3}$ In addition, the crystallinity, stereochemistry, and molecular weights of the polymers also affect their rates of biodegradation. ${ }^{4}$

Poly(L-lactide) (PLLA: Fig. 1C) is a commercially available and well-known biodegradable polymer. ${ }^{5}$ Proteinase K hydrolyses the ester bonds in PLLA although its native substrates are peptides. Proteinase $\mathrm{K}$ predominantly hydrolyses amorphous regions of PLLA, whereas it is difficult to degrade the folded crystals. Therefore, the higher the crystallinity, the slower the enzymatic degradation rate. ${ }^{4 a-e}$ Molecular weight is also a factor that influences the enzymatic degradation rate. UV irradiation has been shown to reduce the molecular weight of PLLA, while increasing the rate of enzymatic degradation. ${ }^{6}$ As a consequence, the term 'control' has been used to mean the 'enhancement or retardation of the total biodegradation rate' in

National Institute of Advanced Industrial Science and Technology (AIST), Tsukuba Central 5, 1-1-1 Higashi, Tsukuba, Ibaraki 305-8565, Japan. E-mail: y.kikkawa@ aist.go.jp; y-norikane@aist.go.jp

$\uparrow$ Electronic supplementary information (ESI) available: Morphological investigations by AFM, additional enzymatic degradation data in PLLA and PCL thin films, and the demonstration of surface patterning. See DOI: $10.1039 / \mathrm{c} 7 \mathrm{ra10598c}$ conventional studies (Fig. 1A). On the other hand, our present study deals with the preparation of biodegradable materials whose enzymatic degradation can be 'switched on' by an external stimulus, rather than materials whose degradation rates can only be controlled (Fig. 1B). To the best of our knowledge, the control of enzymatic-degradation initiation using light has not been reported.

In our previous studies, ${ }^{7,8}$ we developed azo-compounds that can switch between their solid and liquid states upon exposure to light. We showed that these phase-transition materials can be employed as reusable photoresists, ${ }^{8 \boldsymbol{a}}$ reworkable adhesives, ${ }^{\mathbf{8 b}, \boldsymbol{c}}$ optical materials, ${ }^{8 \boldsymbol{d}}$ and for the photo-induced translational motion of crystals. ${ }^{8 e f}$ The characteristic features of our
(A) Conventional studies

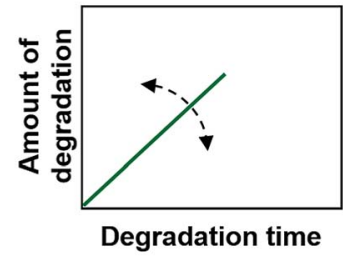

(B) Our present study

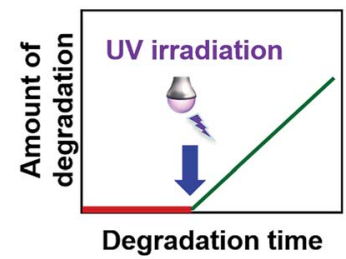

(C) PLLA<smiles>CCCC(=O)[C@@H](C)OCC</smiles>

(D) Azo

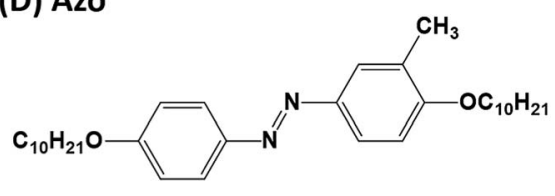

Fig. 1 Schematic diagrams illustrating concepts for controlling enzymatic degradation in (A) conventional studies and (B) our present study. In $(A)$ the degradation rate (slope of the green line) is tuned, whereas in (B) (our study) degradation is initiated and controlled by UV irradiation (at the time indicated by the blue arrow). (C) Chemical structures of PLLA and (D) 4,4'-didecyloxy-3-methylazobenzene (Azo) used in this study. 


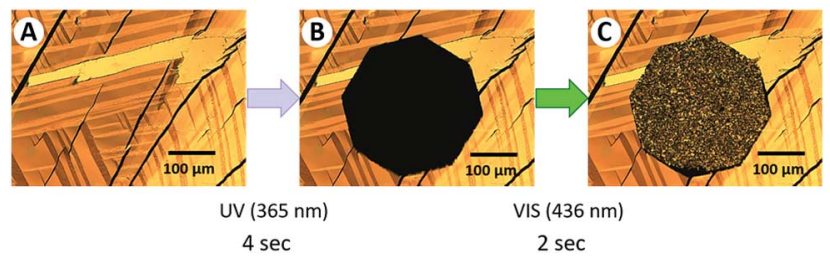

Fig. 2 Optical micrographs of Azo films prepared on glass substrates. (A) As prepared, (B) UV irradiated at $365 \mathrm{~nm}$ for $4 \mathrm{~s}$ in the octagonal area; and (C) exposure to visible light at $436 \mathrm{~nm}$ for $2 \mathrm{~s}$ following UV irradiation

azo-compound $\left(4,4^{\prime}\right.$-didecyloxy-3-methylazobenzene, ${ }^{\text {sa, }}$ Azo: Fig. 1D) are shown in Fig. 2. UV irradiation $(365 \mathrm{~nm})$ in the octagonal region initiates the melting of Azo, which is evidenced by the loss of birefringence in its optical microscope image. Irradiation with visible light $(436 \mathrm{~nm})$ led to recrystallization of the molten Azo and the recovery of its birefringence.

In this contribution, we fabricate a biodegradable polymeric material whose enzymatic degradation can be initiated by irradiation with light. The photo-responsive Azo material is complexed with a biodegradable polymer; in other words, Azo is coated on the PLLA surface to form a bilayer thin film. The enzymatic degradation of the polymer by proteinase $\mathrm{K}$ is regulated and initiated by the phase transition exhibited by Azo; the enzymatic-degradation behaviour of the bilayer thin film was mainly evaluated by atomic force microscopy (AFM).

\section{Experimental}

\section{Preparation of PLLA thin films}

PLLA was purchased from Polysciences Inc. (Warrington, PA), and its number-averaged molecular weight $\left(M_{\mathrm{n}}\right)$ and polydispersity $\left(M_{\mathrm{w}} / M_{\mathrm{n}}\right)$ were determined by gel permeation chromatography (GPC) at $40{ }^{\circ} \mathrm{C}$ using a Shimazu 10A GPC system fitted with a $6 \mathrm{~A}$ refractive index detector; Shodex K-80M and K802 columns were jointly used with chloroform as the eluent. The $M_{\mathrm{n}}$ and $M_{\mathrm{w}} / M_{\mathrm{n}}$ of PLLA were determined to be $420 \mathrm{kDa}$ and 1.7 , respectively. The PLLA was dissolved in chloroform at a concentration of $1.0 \%(\mathrm{w} / \mathrm{v})$, and spin-cast onto a silicon substrate $\left(10 \times 10 \mathrm{~mm}^{2} ; 4000 \mathrm{rpm}\right.$ for $\left.1 \mathrm{~min}\right)$ pre-cleaned by treatment with UV-ozone (UV253, Filgen) for $30 \mathrm{~min}$. The $\sim 70 \mathrm{~nm}$-thick cast thin film was melted at $220^{\circ} \mathrm{C}$ and quenched at $0{ }^{\circ} \mathrm{C}$; as a result, a smooth amorphous PLLA thin film was prepared. ${ }^{9}$

\section{Coating by Azo and UV irradiation}

Azo, prepared as reported previously, ${ }^{8 a, c}$ was dissolved in heptane at a concentration of $1.0 \%(\mathrm{w} / \mathrm{v})$, and spin-cast onto the PLLA amorphous thin film at a rotation speed of $3000 \mathrm{rpm}$ for $1 \mathrm{~min}$. To improve the adhesion between PLLA and Azo, the amorphous PLLA thin film was pre-treated with UV-ozone for $30 \mathrm{~min}$. A ring-shaped mask (frame width: $1 \mathrm{~mm}$; hole diameter: $5 \mathrm{~mm}$ ) was placed on the bilayer thin film, and the film was UV irradiated to induce the solid-to-liquid phase transition of Azo
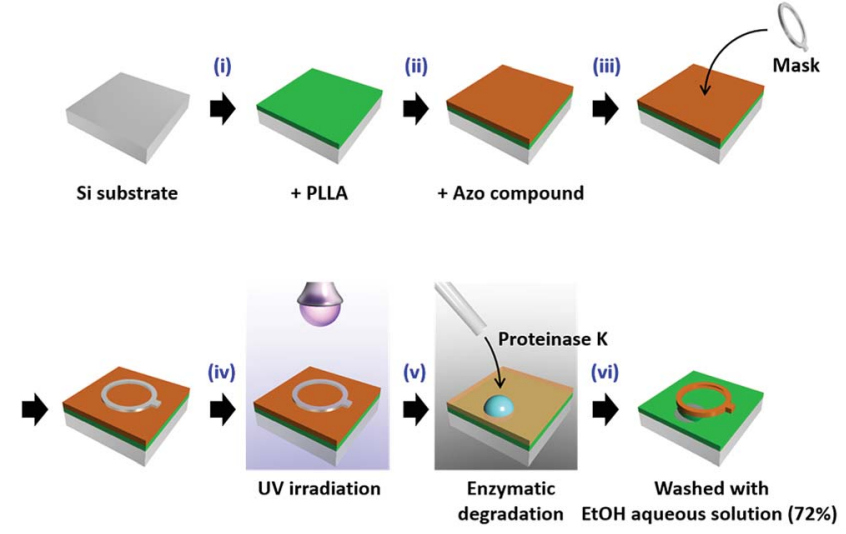

Scheme 1 Schematic diagram illustrating the preparation of the thin film, UV irradiation, and enzymatic degradation. (i and ii) A PLLA thin film was prepared on a Si substrate and coated by Azo to produce a bilayer thin film by spin casting. (iii, iv) A ring-shaped mask was placed on the thin film after which it was UV irradiated (365 nm) for $1 \mathrm{~min}$. (v) A buffer solution containing proteinase $\mathrm{K}$ was deposited on the thin film in the dark and enzymatic degradation was carried out for a given time. (vi) The thin film was finally washed with aqueous ethanol and water.

(365 nm LED, Asahi Spectra Inc. POT-365, $100 \mathrm{~mW}$ ). The detailed experimental set-up is shown in Scheme 1.

\section{Enzymatic degradation by proteinase $\mathrm{K}$}

Proteinase $\mathrm{K}$ was purchased form Roche Diagnostics (Mannheim, Germany), and dissolved in $50 \mathrm{mM}$ Tris-HCl buffer (pH 8.5) such that a $1 \mathrm{mg} \mathrm{mL}{ }^{-1}$ solution was obtained. A droplet of the enzyme solution was deposited on the bilayer thin film and allowed to stand for 5-20 min at room temperature $\left(25^{\circ} \mathrm{C}\right)$. The thin film surface was then washed with aqueous ethanol $(72 \% \mathrm{v} / \mathrm{v})$ and pure water (three times); the residual solvent was removed with $\mathrm{N}_{2}$ gas, and dried at under ambient conditions.

\section{Morphological investigations}

The surface morphology of each PLLA thin film was examined by AFM (SPI4000/SPA400, Hitachi High-Technologies, Japan). An OMCL-AC240TS-W2 cantilever (Olympus, Japan) was used in dynamic-force (tapping) mode. Several sets of $50 \times 50 \mu \mathrm{m}$ areas at different positions on each film were examined in order to determine the depth of erosion following enzymatic degradation.

\section{Results and discussion}

The experimental procedure, shown in Scheme 1, involves the following steps: ( $i$ and ii) a thin film of PLLA was prepared on a Si substrate and coated with Azo by spin casting to form a bilayer thin film. (iii and iv) A ring-shaped mask was placed over the bilayer thin film, after which it was irradiated by UV light $(365 \mathrm{~nm}$ ) for $1 \mathrm{~min}$. Following this procedure, we expected the region under the mask to remain unchanged, whereas the Azo layer in the UV-light-exposed area was expected to have melted. (v) After removing the mask, a droplet of proteinase $\mathrm{K}$ in 
buffer solution was deposited directly onto the film such that it covered the region originally protected by the mask, as well as surrounding areas. (vi) The thin film was then washed with aqueous ethanol, resulting in the removal of the molten Azo and the degradation products eluted by the enzyme solution. Steps (iii-vi) were carried out in the dark. Each enzymatic-degradation experiment was conducted with a different sample of the thin film for a different degradation time.

Surface morphologies of the thin films were examined by AFM (see ESI $\dagger$ ); each film exhibited a smooth surface with a roughness $\left(R_{\mathrm{a}}\right)$ of $0.3 \mathrm{~nm}$ (Fig. $\left.\mathrm{S} 1 \mathrm{~A} \dagger\right)$. After Azo was spin-cast onto the PLLA surface, crystalline materials of around $1 \mu \mathrm{m}$ in size were observed, resulting in increased surface roughness (Fig. S1B $\dagger$ ). After irradiation with UV (365 nm) and then visible $(436 \mathrm{~nm})$ light, layers with larger crystals (over $5 \mu \mathrm{m}$ in size) were observed, indicating that the Azo had melted and recrystallized on the PLLA surface. This result indicates that the Azo covers the entire PLLA surface, and that UV irradiation is capable of inducing Azo melting even on the PLLA surface.

The enzymatic-degradation behaviour of the bilayer thin film was examined. Fig. 3A shows a typical photographic image of the bilayer thin film after enzymatic degradation by proteinase $\mathrm{K}$ for $20 \mathrm{~min}$. Three regions with different contrasts are evident in this image. The point at which the three regions with different contrasts intersect, as highlighted by the green square in Fig. 3A, was examined by AFM. The AFM images in Fig. 3B and $\mathrm{C}$ reveal three regions of different heights. The crosssectional profile along the white line in Fig. 3B is shown in Fig. 3D, and reveals height differences of $\sim 50 \mathrm{~nm}$ for each step. According to the experimental procedure, the lowest layer is the Si substrate, the second is the PLLA surface, and top layer corresponds to the Azo surface. Hence, the degradation area is effectively controlled by the combination of UV irradiation and enzymatic degradation.
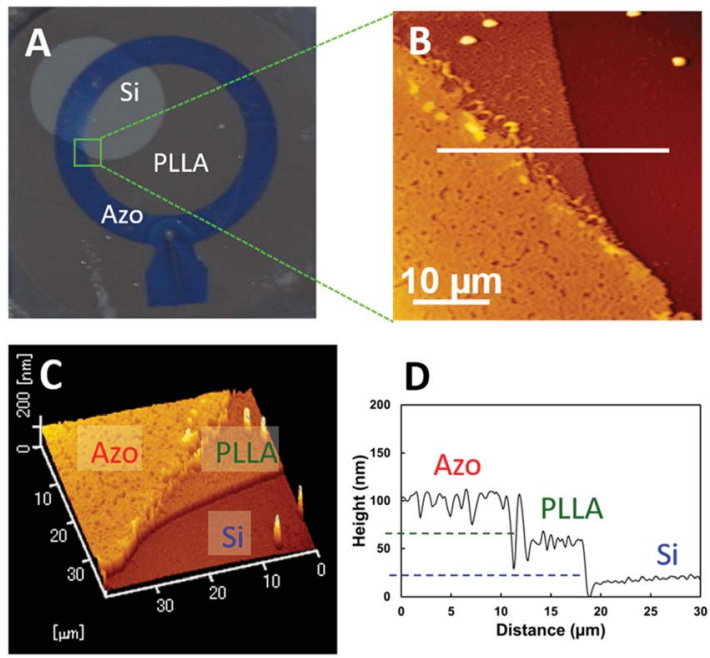

Fig. 3 (A) Photographic image of a bilayer thin film after the treatment shown in Scheme 1. (B) AFM height profile, and (C) three-dimensional profile of the area in the green square in (A). (D) Cross-sectional data along the white line in (B).
In our previous paper, ${ }^{\mathbf{1 0}}$ we reported that weight loss due to enzymatic degradation, measured by quartz-crystalmicrobalance (QCM) and surface-plasmon-resonance (SPR) techniques, is proportional to the erosion depth measured by AFM. Therefore, we propose that the time-dependent measurement of erosion depth can be used to evaluate both the onset and progress of enzymatic degradation.

First, we examined by AFM the enzymatically degraded PLLA thin film devoid of Azo but pre-treated with UV-ozone; timedependent erosion-depth measurements were also performed (Fig. S2 $\dagger$ ). In this experiment, the UV-ozone treatment was applied to the PLLA surface since this treatment is also used during the bilayer-fabrication process; this surface was used as a reference to assess changes in degradation rate. ${ }^{11}$ As shown in Fig. S2, $\dagger \mathrm{a} \sim 70 \mathrm{~nm}$-thick thin film was eroded over a period of 20 min, during which the erosion depth $(d)$ was observed to deepen with increasing degradation time; the erosion depth as a function of degradation time is displayed by the red squares in Fig. 4.

Subsequently, we investigated the erosion depth of the bilayer film composed of both PLLA and Azo. No erosion was observed in the bilayer thin film by AFM prior to UV irradiation, even after the deposition of proteinase $\mathrm{K}$ for $20 \mathrm{~min}$ (flat bluecircle region of Fig. 4); this is because the PLLA surface is covered with Azo that cannot be hydrolysed by proteinase $\mathrm{K}$. However, after UV irradiation, the bilayer thin film was degraded by proteinase K (see ESI $\dagger$ ). To our surprise, the enzymatic degradation of PLLA took place without the removal of the liquid Azo layer. We propose that the buffer solution containing proteinase $\mathrm{K}$ extruded or penetrated into the liquid Azo layer to degrade PLLA. The rate of erosion of the bilayer thin film after UV irradiation was almost identical to that of the PLLA thin film pre-treated with UV light (two slopes in Fig. 4), suggesting that the molten Azo does not interfere with the hydrolytic action of the enzyme.

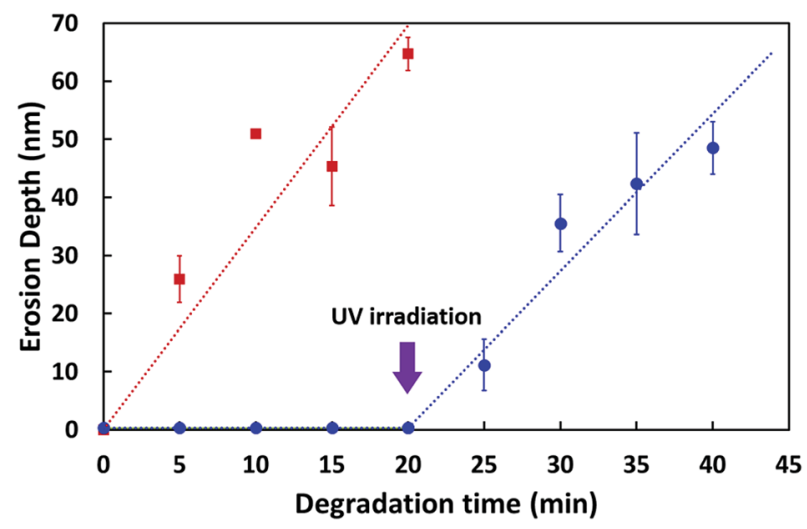

Fig. 4 Erosion depth as a function of degradation time. The red squares are data for the enzymatic degradation of the PLLA thin film treated with UV-ozone, ${ }^{11}$ whereas the blue circles are for the bilayer thin film in which the PLLA started to erode after UV irradiation. The erosion rate was $3.5 \pm 0.2 \mathrm{~nm} \mathrm{~min}{ }^{-1}$ (red line) and $2.7 \pm 0.4 \mathrm{~nm} \mathrm{~min}{ }^{-1}$ (blue line), respectively. A fresh film sample was prepared for each measurement and all measurements were carried out at least three times. The error bars depict standard deviations in the data. 
To explore the generality of the present method for controlling the onset of enzymatic degradation, we also examined another biodegradable polymer, poly( $\varepsilon$-caprolactone) (PCL) with lipase as the enzyme, (see ESI $\dagger$ ). We successfully controlled the initiation of the enzymatic degradation of PCL by the phase transition of Azo induced by exposure to UV radiation.

Through the combination of the mask pattern and the position of the enzyme droplet, a patterned surface was created (ESI, Fig. S6†). It should be noted that the current method does not use harsh reagents such as acids or bases. Hence, we created stimulus-responsive biodegradable films using a simple method; the enzymatic degradation of these films is initiated by UV irradiation.

\section{Conclusions}

Control over the initiation of enzymatic degradation was successfully demonstrated through the use of 4,4'-didecyloxy-3methylazobenzene (Azo) as a surface coating; the solid-to-liquid transition of Azo is controlled by exposure to light. A bilayer thin film was prepared by coating the PLLA thin film with Azo. Erosion depth measurements by AFM revealed that enzymatic degradation by proteinase $\mathrm{K}$ is initiated by exposure to UV light, and that the resulting enzymatic degradation rate was almost identical to that of the original PLLA thin film, suggesting that liquid Azo does not hinder the degradation process. This simple method was also applied to another representative biodegradable polymer, namely PCL.

We believe that the present concept, i.e., stimulus-triggered enzymatic degradation, will contribute to the development of lifetime-controlled biodegradable materials. Moreover, proper masking and subsequent enzymatic degradation allows positional control of surface etching, and provides an environmentally friendly surface-patterning method.

\section{Conflicts of interest}

There are no conflicts to declare.

\section{Acknowledgements}

This work was supported by a Grant-in-Aid for Challenging Exploratory Research (JSPS KAKENHI: Grant Number 26620152), Japan.

\section{Notes and references}

1 (a) S. Doppalapudi, A. Jain, W. Khan and A. J. Domb, Polym. Adv. Technol., 2014, 25, 427; (b) M. J.-L. Tschan, E. Brulé, P. Haquette and C. M. Thomas, Polym. Chem., 2012, 3, 836; (c) A. Kumar, K. Karthick and K. P. Arumugam, Int. J. Chem. Eng. Appl., 2011, 2, 164; (d) G. E. Luckachan and C. K. S. Pillai, J. Polym. Environ., 2011, 19, 637; (e) X. W. Zhao, L. Wang and D. Liu, J. Chem. Technol. Biotechnol., 2007, 82, 1115.

2 (a) B. Laycock, M. Nikolić, J. M. Colwell, E. Gauthier, P. Halley, S. Bottle and G. George, Prog. Polym. Sci., 2017,
71, 144; (b) T. Hiraishi and S. Taguchi, Mini-Rev. Org. Chem., 2009, 6, 44; (c) D. Jendrossek, Extracellular polyhydroxyalkanoate depolymerases: The key enzymes of PHA degradation, in Biopolymers, ed. Y. Doi and A. Steinbüchel, Wiley-VCH Verlag GmbH, Weinheim, 2002, vol. 3b, pp. 41-83; (d) K. Sudesh, H. Abe and Y. Doi, Prog. Polym. Sci., 2000, 25, 1503.

3 (a) Y. Ikada and H. Tsuji, Macromol. Rapid Commun., 2000, 21, 117; (b) C. S. Ha and W. J. Cho, Prog. Polym. Sci., 2002, 27, 759; (c) H. Tsuji, Macromol. Biosci., 2005, 5, 569; (d) S. Wang, W. Cui and J. Bei, Anal. Bioanal. Chem., 2005, 381, 547; (e) R. M. Rasal, A. V. Janorkar and D. E. Hirt, Prog. Polym. Sci., 2010, 35, 338; (f) S. S. Ray, Acc. Chem. Res., 2012, 45, 1710.

4 (a) M. S. Reeve, S. P. McCarthy, M. J. Downey and R. A. Gross, Macromolecules, 1994, 27, 825; (b) R. T. MacDonald, S. P. McCarthy and R. A. Gross, Macromolecules, 1996, 29, 7356; (c) S. Li and S. McCarthy, Macromolecules, 1999, 32, 4454; (d) H. Tsuji and S. Miyauchi, Polym. Degrad. Stab., 2001, 71, 415; (e) H. Tsuji and S. Miyauchi, Polymer, 2001, 42, 4463; $(f)$ S. Li, M. Tenon, H. Garreau, C. Braud and M. Vert, Polym. Degrad. Stab., 2000, 67, 85; $(g)$ H. Tsuji and S. Miyauchi, Biomacromolecules, 2001, 2, 597.

5 (a) M. Singhvi and D. Gokhale, RSC Adv., 2013, 3, 13558; (b) H. Tsuji, Polylactides, in Biopolymers, Y. Doi and A. Steinbüchel, Wiley-VCH Verlag GmbH, Weinheim, 2002, vol. 4, pp. 129-177; (c) T. Iwata, H. Abe and Y. Kikkawa, Enzymatic degradation, in Poly(lactic acid): synthesis, structures, properties, processing, and applications, ed. R. A. Auras, L. T. Lim, S. E. Selke and H. Tsuji, John Wiley \& Sons, Inc., 2010, pp. 383-399.

6 (a) H. Tsuji, Y. Echizen and Y. Nishimura, J. Polym. Environ., 2006, 14, 239; (b) H. Tsuji, K. Shimizu and Y. Sato, J. Appl. Polym. Sci., 2012, 125, 2394.

7 (a) Y. Norikane, Y. Hirai and M. Yoshida, Chem. Commun., 2011, 47, 1770; (b) M. Hoshino, E. Uchida, Y. Norikane, R. Azumi, S. Nozawa, A. Tomita, T. Sato, S. Adachi and S. Koshihara, J. Am. Chem. Soc., 2014, 136, 9158.

8 (a) Y. Norikane, E. Uchida, S. Tanaka, K. Fujiwara, E. Koyama, R. Azumi, H. Akiyama, H. Kihara and M. Yoshida, Org. Lett., 2014, 16, 5012; (b) H. Akiyama, S. Kanazawa, Y. Okuyama, M. Yoshida, H. Kihara, H. Nagai, Y. Norikane and R. Azumi, ACS Appl. Mater. Interfaces, 2014, 6, 7933; (c) Y. Norikane, E. Uchida, S. Tanaka, K. Fujiwara, H. Nagai and H. Akiyama, J. Photopolym. Sci. Technol., 2016, 29, 149; (d) E. Uchida, K. Sakaki, Y. Nakamura, R. Azumi, Y. Hirai, H. Akiyama, M. Yoshida and Y. Norikane, Chem.-Eur. J., 2013, 19, 17391; (e) E. Uchida, R. Azumi and Y. Norikane, Nat. Commun., 2015, 6, 7310; ( $f$ ) Y. Norikane, S. Tanaka and E. Uchida, CrystEngComm, 2016, 18, 7225.

9 (a) Y. Kikkawa, M. Fujita, H. Abe and Y. Doi, Biomacromolecules, 2004, 5, 1187; (b) K. Yamashita, Y. Kikkawa, K. Kurokawa and Y. Doi, Biomacromolecules, 2005, 6, 850.

10 (a) Y. Kikkawa, M. Fukuda, N. Ichikawa, A. Kashiwada, K. Matsuda, M. Kanesato and T. Hiraishi, J. Mater. Chem. 
A, 2013, 1, 4667; (b) Y. Shinozaki, Y. Kikkawa, S. Sato, T. Fukuoka, T. Watanabe, S. Yoshida, T. Nakajima-Kambe and H. K. Kitamoto, Appl. Microbiol. Biotechnol., 2013, 97, 8591.
11 The rate of enzymatic degradation of a PLLA thin film treated with UV ozone was slower compared to that of the pristine PLLA thin film (ESI $\dagger$ ). A detailed study was reported in our previous paper (ref. 10a). 